http://dx.doi.org/10.35381/r.k.v5i1.784

\title{
Aprendizaje Cooperativo, Powtoon y desarrollo de destrezas en inglés
}

\section{Cooperative Learning, Powtoon and development of english Skills}

\author{
Marcia Geoconda González-González \\ marcia.gonzalez@psg.ucacue.edu.ec \\ Universidad Católica de Cuenca, Azogues \\ Ecuador. \\ https://orcid.org/0000-0002-2432-1015 \\ Darwin Gabriel García-Herrera \\ dggarciah@ucacue.edu.ec \\ Universidad Católica de Cuenca, Azogues \\ Ecuador \\ https://orcid.org/0000-0001-6813-8100 \\ Nancy Marcela Cárdenas-Cordero \\ ncardenasc@ucacue.edu.ec \\ Universidad Católica de Cuenca, Azogues \\ Ecuador \\ https://orcid.org/0000-0002-6250-6504 \\ Juan Carlos Erazo-Álvarez \\ jcerazo@ucacue.edu.ec \\ Universidad Católica de Cuenca, Cuenca \\ Ecuador \\ https://orcid.org/0000-0001-6480-2270
}

Recibido: 24 de abril de 2020

Revisado: 17 de mayo de 2020

Aprobado: 11 de junio de 2020

Publicado: 29 de junio de 2020 


\title{
RESUMEN
}

El objetivo del presente trabajo investigativo fue demostrar como incide el aprendizaje cooperativo con su técnica de rompecabezas combinada con la tecnología mediante el uso de la aplicación Powtoon en el desarrollo de las destrezas del idioma Inglés. Metodológicamente se desarrolló mediante un tipo descriptiva. La aplicación Powtoon una herramienta con la que los estudiantes se sienten cómodos para trabajar y mejoren sus destrezas. Esta aplicación diseña otra forma de aprender el idioma Inglés, refuerza sus capacidades de inventar, crear y aplicar su propia creatividad para presentar el trabajo de mejor manera, les permite crear su propio conocimiento, pero siempre en base a conocimientos previos y de acuerdo a una planificación establecida en la cual exista la interacción del profesor - estudiante para la construcción de un aprendizaje significativo sin olvidar la retroalimentación luego de cada presentación grupal.

Descriptores: Aprendizaje activo; autoaprendizaje; experimento educacional; idioma de enseñanza. (Palabras tomadas del Tesauro UNESCO).

\begin{abstract}
The objective of this research work was to demonstrate how cooperative learning affects its puzzle technique combined with technology through the use of the Powtoon application in the development of English language skills. Methodologically it was developed using a descriptive type. The Powtoon application a tool with which students feel comfortable to work and improve their skills. This application designs another way to learn the English language, reinforces their abilities to invent, create and apply their own creativity to present the work in a better way, allows them to create their own knowledge, but always based on previous knowledge and according to a established planning in which there is teacher-student interaction for the construction of meaningful learning without forgetting the feedback after each group presentation.
\end{abstract}

Descriptors: Activity learning; self instruction; educational experiments; language of instruction. (Words taken from the UNESCO Thesaurus). 


\section{INTRODUCCIÓN}

El idioma Inglés ha ido ganando un lugar muy importante en el desarrollo profesional de las personas y es necesario por ende desarrollar las destrezas de saber escuchar, escribir, leer y hablar para enfrentar a un mundo globalizado en el cual. la tecnología se combina para el aprendizaje de este idioma. (Robayo-Guerrero, 2019) adquirir una competencia en el lenguaje oral se debe a la forma como se ha desarrollado estas destrezas del idioma en los estudiantes apoyados en la tecnología y sus aplicaciones que nos ofrecen hoy en día para mejorar la enseñanza- aprendizaje.

Considerando que el aprendizaje del idioma Inglés se da desde tempranas edades, es posible plantear nuevas estrategias enfocadas a disfrutar y mejora este proceso, como por ejemplo el aprendizaje cooperativo en el cual logra que el estudiante se involucre en su adquisición del conocimiento mediante trabajos en grupos pequeños en los mismo que se reflejaran el esfuerzo por recolectar la información, analizarla, discernir y llegar a un consenso para lograr un aprendizaje significativo apoyado de sus compañeros de clase (Borregales, 2020).

Según (Loor-Salmon, Palma-Villavicencio, Saltos-Rodriguez, \& Bolívar-Chavez, 2018) los docentes que utilizan el método tradicional en la enseñanza del idioma no motivan sus clases ya que se dedican solo a repeticiones de vocabulario o completar espacios en blanco entre otras actividades monótonas en cambio al incorporar el trabajo cooperativo a este proceso será más fácil calificar las actividades grupales o lecciones orales. El objetivo no será solo de incorporar el trabajo cooperativo al desarrollo del aprendizaje, sino de fusionar las habilidades que poseen en el majeo de la tecnología para mejorar sus destrezas en el idioma (Briceño-de-Osorio, 2019).

Como se puede apreciar el idioma Inglés tiene mucha relevancia tanto en el campo estudiantil como en el profesional creando competencias lingüísticas a lo largo del desarrollo integral de la persona, (lleana de León Sac, 2013) más ahora que el idioma se ha globalizado y que la tecnología está en auge es necesario que los estudiante dominen las destrezas comunicativas, (leer, escuchar, escribir y hablar) llamadas lingüísticocomunicativa que marcarán la educación del siglo XXI (Fernando, 2015). 
(Namaziandost, Shatalebi, \& Nasri, 2019), (Rusyda \& Supari, 2019) y (Braun, 2017) coindicen que la adquisición de destrezas en el idioma Inglés se debe mucho al factor motivación por parte del profesor en clases, los estudiantes deben compartir un ambiente cómodo y seguro para que se dé el aprendizaje en especial de Inglés, se debe fomentar el interés por aprender mediante varias aplicaciones que la tecnología brinda y de esta manera contribuir a potenciar las destrezas con aprendizajes significativos (Dovale, 2018).

Ante todo, lo expuesto, verdad es que el idioma ingles es necesario en todos los ámbitos en especial educativo, el problema se da en estudiantes de segundo de bachillerato de la Unidad Educativa Particular La Providencia quienes a pesar de recibir durante toda su vida estudiantil la enseñanza del idioma como una asignatura con 5 horas a la semana, no están lo suficientemente aptos para desarrollar sus destrezas en Inglés. Lo que se demuestra través de sus notas en especial en pruebas sumativas un $66 \%$ de 131 estudiantes tienen una nota menor de 7 y no satisfacen a las expectativas del idioma ni del profesor.

El problema está presente, razón por la cual este trabajo investigativo propone una nueva forma de enseñar el idioma utilizando el aprendizaje cooperativo, apoyado en la tecnología en especial a la aplicación Powtoon como herramienta para el desarrollo de trabajo en grupo, lo que podría ayudar al estudiante a sentirse motivados por aprender el idioma de una forma diferente dejando a lado lo tradicional del idioma (Arroyo-Carrera, 2020).

Por otro lado, (Torres-Cajas, 2017) manifiesta que durante la educación tradicional, el profesor era quien ejercía su cátedra frente a sus estudiantes quienes se limitaban a ser entes pasivos y receptores de conocimiento profesor-estudiante, sin embargo, esta situación ha ido cambiando para dar paso al protagonismo del estudiante en este proceso educativo generando de esta manera una interacción más directa profesor-estudianteestudiante en un contexto social y real, por cuanto todos los seres humanos son parte de un contexto y por consiguiente nadie aprende solo, sino a base de las experiencia compartidas por los demás. 
El aprendizaje cooperativo no es nuevo por lo contrario data desde el siglo XVII con Commenius (1592-1670) quien aseguraba que este trabajo ayudaba a los estudiantes a aprender juntos es decir se enseñaban entre ellos (Pujolàs Maset, Pere, 2003), y desde allí hasta la fecha muchos son los establecimientos que utilizan este trabajo cooperativo en algunas asignaturas enfocado a diferentes objetivos y en muchas partes. Autores como (Loor-Salmon, Palma-Villavicencio, Saltos-Rodriguez, \& Bolívar-Chavez, 2018) dan una clara definición del aprendizaje cooperativo como una actividad estructurada de pequeños grupos que intercambian información social que comparten las mismas responsabilidades de su aprendizaje motivados por aprender el uno del otro.

Según (Torres-Cajas, 2017) resultados positivos sobre el uso del aprendizaje cooperativo se evidencia desde hace más de 20 años el trabajo cooperativo potencializa el sentido de competencia sana y agudiza el esfuerzo de los estudiantes por lograr un aprendizaje a largo plazo con un nivel amplio de razonamiento. Es decir, un aprendizaje que mejora las habilidades cognitivas dentro de un grupo de trabajo heterogéneo, pero con un mismo objetivo en común.

Por otro lado, la tecnología está al servicio de la educación para dar un matiz de innovador a este aprendizaje cooperativo al usar los dispositivos móviles con sus aplicaciones ya que brinda la posibilidad de interactuar sin importar el lugar ni la hora ni el momento por su conectividad, portabilidad, movilidad y accesibilidad (Sung, Chang \& Liu, 2016). O también se puede utilizar aplicaciones de office como el Power Point, Word, entre otras que facilitan el aprendizaje del idioma dar la oportunidad a los estudiantes de diseñar su propia forma de aprendizaje interdisciplinario.

Con todos estos antecedentes el objetivo del presente trabajo investigativo fue demostrar como incide el aprendizaje cooperativo con su técnica de rompecabezas combinada con la tecnología mediante el uso de la aplicación Powtoon en el desarrollo de las destrezas del idioma Inglés. 


\section{Referencial teórico}

\section{Aprendizaje cooperativo estructurado}

Es llamado así por (Kagan, 2013) después de varias investigaciones acerca de este aprendizaje cooperativo y citado en (Lemos-Rodriguez, 2018), quien manifiesta que es estructurado porque se trata de una serie de juegos con sus propias reglas fáciles de aprender y jugar con un resultado flexible y efectivo, también (García-Sánchez, 2015) manifiesta que realizar un trabajo cooperativo despierta la motivación, reduce el stress y desarrolla actividades positivas frente al aprendizaje como respeto y confianza.

Sin embargo, (Torres-Cajas, 2017) lo considera como una estrategia bien organizada y preparada por el docente y los estudiantes para trabajar con grupos heterogéneos de forma interdependiente con un mismo objeto de lograr un aprendizaje significativo y el éxito en la tarea asignada. Si bien es cierto que el ser humano es una persona social razón de más para aprender juntos el uno del otro.

Ante lo expuesto es los párrafos anteriores se puede estar hablando ya de características del aprendizaje cooperativo siendo estas entre las más importantes: Según (LoorSalmon, Palma-Villavicencio, Saltos-Rodriguez, \& Bolívar-Chavez, 2018)

1. Se llega a lograr el objetivo del trabajo.

2. Mejora las relaciones interpersonales entre los estudiantes.

3. Desarrollo valores humano.

4. Reemplaza la competencia por cooperación.

Así mismo, (Martínez-Lirola, 2017), coinciden con estas características, pero mencionadas de otra manera:

1. Trabajo mutuo: los integrantes del grupo comparten el mismo objetivo y materiales.

2. Responsabilidad compartida: cada integrante es el que determina el fracaso o el éxito $d$ todos.

3. Relaciones interpersonales: comparten la información mejora la comunicación.

4. Aprenden a escucharse desarrollo de actitudes, valores entre ellos para resolver problemas o buscar la mejor solución.

5. Valoran el trabajo en grupo aprenden juntos. 
Además, en ambas citaciones se verifica que existe cambio de roles dentro de este proceso el profesor pasa a ser un coordinador o director del trabajo y los estudiantes son quienes se encargan de la adquisición de su propio conocimiento disfrutando en grupo y mejorando la comunicación. De esta manera el aprendizaje cooperativo busca fortalecer las destrezas en inglés, por cuanto si las instrucciones creadas son muy bien enfocadas y estructuradas se replantea potenciar las destrezas de speaking, listening, reading y writing y crear un ambiente de cooperación en vez de competencia. Si se toma de esta manera, el aprendizaje cooperativo es otra forma de pensar a cerca de un trabajo en un grupo tradicional, es una propuesta más compleja en la misma que prevalece el aprender mutuamente y respetar las diferencias existentes dentro de un mismo grupo. Este aprendizaje cooperativo también tiene sus técnicas y entre las más utilizadas tenemos las siguientes:

1.- TAI; Team Assisted Indivudualization. - En esta técnica el trabajo es individual con un mismo tema a trabajar siguiendo reglas específicas un aprendizaje individualizado ajustado a las necesidades de cada estudiante.

2.- La tutoría entre iguales Peer tutoring. - un aprendizaje cooperativo entre pares, el uno es el tutor y el otro el estudiante.

3.- El rompecabezas Jigsaw. - Un aprendizaje cooperativo de grupos pequeños con fragmentos de un tema principal.

4.- Los grupos de investigación- group investigación. - Un aprendizaje cooperativo más complejo conocido como el trabajo por proyectos. (Pujolàs Maset, Pere, 2003)

Técnica rompecabezas Jigsaw

(Hamzah-Alamri, 2018) expresan que es una técnica diseñada por Eliot Aronson en 1971 después de descubrir que los métodos tradicionales crea en los estudiantes una competitividad desfavorable y en consecuencia un ambiente contrario dentro de clases por que no están motivados para aprovechar y desarrollar destrezas en este aprendizaje, en cambio al implementar el rompecabezas como un aprendizaje cooperativo ayuda a los estudiantes a sentirse dispuestos por aprender, desarrollar las destrezas interpersonales y alcanzar sus metas. 
Se le denomina de esta manera porque cada estudiante es una pieza fundamental e importante de esta técnica para armar el tema o lección asignada, los estudiantes se convertirán en expertos después de formar parte un vínculo cooperativo grupal por la sencilla razón que tienen que analizar, discernir, diseñar y muchas veces memorizar la información y el éxito o fracaso del grupo dependería del grado de involucramiento de sus miembros (Universa, 2020).

Es una técnica muy fácil de implementar en el aula de clase al seguir los siguientes pasos: (FundaciónMapfre, 2020)

1.- Dividir en pequeños heterogéneos grupos de trabajos de 4 a 5 integrantes.

2.- Nombrar a un responsable del grupo quien se responsabilice del trabajo.

3.- Dividir el contenido de la clase en subtemas.

4.- Designar los subtemas a cada grupo.

5.- Establecer el tiempo en desarrollar el trabajo designado.

6.- Escoger la información que será presentada.

7.- Durante la presentación incentivar a los compañeros hacer preguntas sobre el tema.

8.- Presentación de los diferentes grupos.

9.- El docente será el coordinador y quía para el trabajo revisando grupo por grupo el trabajo.

10.- Evaluación de la tarea o trabajo en grupo para dar importancia.

El producto de esta técnica puede ser expuesta mediante presentaciones realizadas en los laboratorios de Inglés en los establecimientos que lo posean, o también se puede ayudar con la tecnología y las diferentes herramientas que están a disponibilidad de los estudiantes siendo el Powtoon una de ellas. De esta manera el docente innovará el aprendizaje tradicional, primero con una técnica flexible y recomendada que es el rompecabezas (Jigsaw) dentro del aprendizaje cooperativo, la misma que dirigida con actividades estratégicas desarrolla las destrezas de Inglés reading (leer), listening (escuchar), writing (escritura) y speaking (hablar).

Powtoon es una herramienta de la web que sirve para realizar presentaciones dinámicas 
de videos creados mediante varias opciones como insertar imágenes, objetos, texto, música e incluso grabar sus voces, Una herramienta fácil de utilizar, el único problema es que se necesita internet para trabajar en ello. (SourceForge, 2020)

Por consiguiente, con este aprendizaje cooperativo, técnica adecuada y junto a los beneficios de la tecnología, los estudiantes se sentirán motivados y su actitud frente al aprendizaje de Inglés será diferente, disfrutar de su aprendizaje, potenciar sus destrezas salir de lo monótono y mejorar su rendimiento académico.

\section{MÉTODO}

Este presente trabajo investigativo experimental está compuesto un grupo de 61 estudiantes de Segundo de bachillerato de la Unidad Educativa La Providencia que trabajaron con el método cooperativo, técnica del rompecabezas y con la aplicación Powtoon para la presentación del producto final (Siguenza-Peñafiel, Erazo-Álvarez, \& Narváez-Zurita, 2020). Luego de cada presentación se realizó un refuerzo en línea debido a que el Ecuador está pasando por una emergencia sanitaria a causa del COVID-19.

Además, es una investigación tipo descriptiva, se empleó una encuesta que tenía 10 preguntas para conocer la posición actitudinal del estudiante frente a la materia de Inglés reflejados en una plataforma llamada Idukay, mediante sus notas de fin de unidad que fue el día 03-05-2020.

Además, tiene una cohorte transversal porque el muestreo fue recolectado una sola vez al final. Se aplicó una metodología llamada SAPIE, (García-Herrera, et al., 2018), la misma que contiene los siguientes pasos: escoger o seleccionar, analizar, planificar, implementar, evaluar que mejoran la comprensión lectora (reading) y expresiva (speaking) las mismas estrategias que ayudaron a complementar para desarrollar las destrezas de escritura (writing) y de escuchar (listening).

Los estudiantes, luego de haber sido seleccionado el contenido por parte del docente, se subdividió en temas cortos que los grupo tenían que desarrollar, cada grupo tenía un coordinador, quien se encargaba de motivar a los integrantes, los grupos fueron estratégicamente seleccionados por parte del profesor, los subtemas fueron escogidos 
por los coordinadores, el siguiente paso fue investigar sobre el subtema específico que les toco a través de las fuentes que proporciona la tecnología, con el objetivo de trabajar sobre unas tres preguntas creadas por el docente acerca del tema.

Luego que se procedió a la recolección de información, análisis y selección de la misma se planificó las posibles formas de presentar el trabajo grupal, siendo en primer lugar el Power Point una herramienta que se trabajó en el laboratorio siempre dirigida o controlado por el docente, pero cabe recalcar que al realizar este trabajo de observó que los estudiantes no dominan estas herramientas de Microsoft, por último fue transformar de Power Point a un creación propia utilizando la aplicación de Powtoon.

Al realizar este trabajo los estudiantes aplicaron sus conocimientos previos y fueron capaces de construir su propio aprendizaje mediante la técnica del rompecabezas y el trabajo cooperativo aplicado dentro del grupo les llevaron a ser solidarios, respetuosos al escucharse las distintas opiniones en base a un solo tema, y la diversidad que existía en el grupo es decir se manifestó valores rescatados en grupo como lo expone MartínezLirola (2017) que mediante la cooperación se recalca la educación en valores adquiriendo competencias para llegar a resolver un problema de trabajo.

La muestra empleada fue aleatoria simple ya que se eligió 2 grupos de un mismo nivel, 61 estudiantes de la Unidad Educativa Particular "La Providencia" de segundo de bachillerato "A" y "C". El colegio está ubicado en la cuidad de Azogues provincia del Cañar, mixto con más de 100 años de funcionamiento y con un número de 1692 estudiantes y cuenta con 62 docentes, 18 administrativos, 5 religiosas y 7 de apoyo.

El instrumento que se utilizó fue una encuesta a través de google drive de 10 preguntas, pero para el análisis solo se escogieron 4 al grupo experimental de 61 estudiantes las que fueron validadas en el software SPSS con una fiabilidad en Alfa de Cronbach de 0,886 . 


\section{RESULTADOS}

\section{Tabla 1}

Prueba T Student

\begin{tabular}{lccccc} 
& \multicolumn{3}{c}{ Valor de prueba $=0$} \\
& T Gl & $\begin{array}{c}\text { Sig. } \\
\text { (bilateral) }\end{array}$ & $\begin{array}{c}\text { Diferencia de } \\
\text { medias }\end{array}$ & $\begin{array}{c}95 \% \text { Intervalo de } \\
\text { confianza para la } \\
\text { diferencia } \\
\text { Inferior }\end{array}$ & Superior \\
\hline $\begin{array}{l}\text { Es importante el cambio de } \\
\text { metodología para disfrutar del } 34,00460 \\
\text { aprendizaje. }\end{array}$ & 0,000 & 3,918 & 3,69 & 4,15 \\
\hline
\end{tabular}

Fuente: Elaboración propia.

Prueba de hipótesis:

$\mathrm{HO} \rightarrow$ Si es importante el cambio de metodología para disfrutar del aprendizaje.

$\mathrm{H} 1 \rightarrow$ No es importante el cambio de metodología para disfrutar del aprendizaje.

Como se puede apreciar en esta tabla 1 se comprueba la hipótesis nula: que es importante el cambio de metodología para un cambio de actitud frente al aprendizaje del idioma. El aprendizaje cooperativo pertenece a una metodología activa, que fue empleada para que se consiga este cambio en los estudiantes.

Se acepta $\mathrm{H} 0$ y se rechaza $\mathrm{H} 1$. 
Marcia Geoconda González-González; Darwin Gabriel García-Herrera; Nancy Marcela Cárdenas-Cordero; Juan Carlos Erazo-Álvarez

\section{Tabla 2}

Tabla de contingencia trabajo en relación con el uso de metodologías

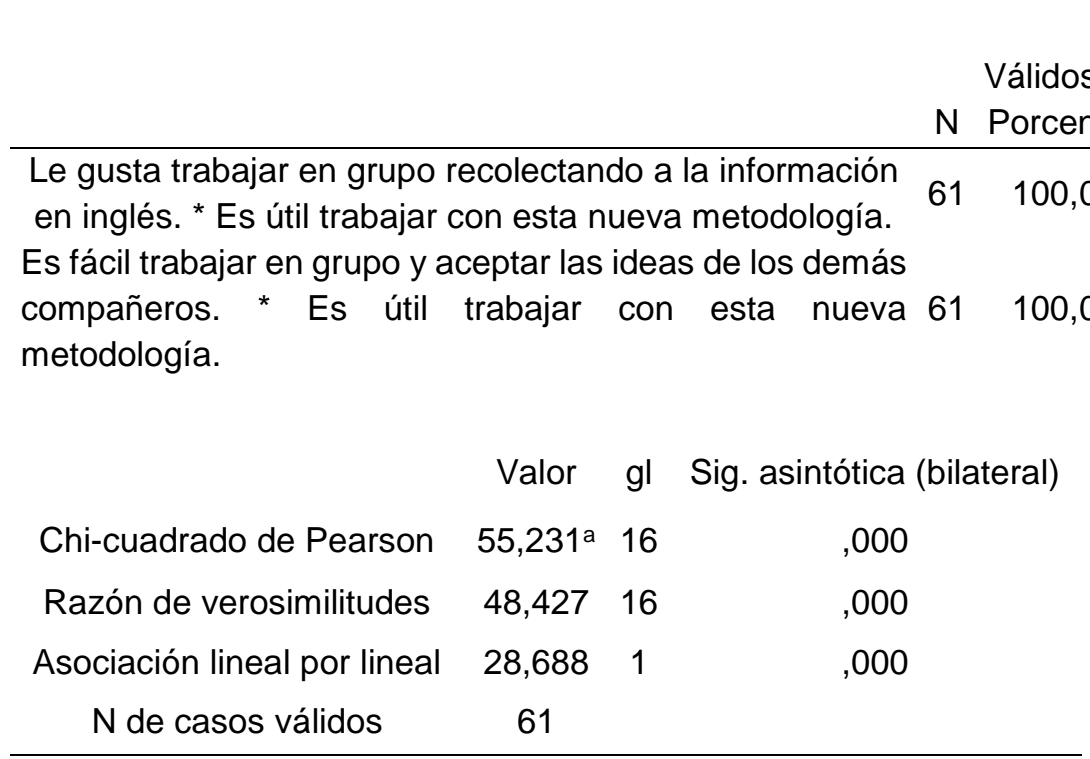

Fuente: Elaboración propia.

H0: Que el trabajar en grupo recolectando información y aceptar las ideas de los demás integrantes si influye al trabajar con esta nueva metodología.

H1: Que el trabajo en grupo recolectando información y el aceptar las ideas de los demás integrantes del grupo no influye al trabajar con esta metodología

En la siguiente tabla 2 se aplica la hipótesis nula que tanto trabajar en grupo recolectando información en Inglés, como aceptar las ideas de los demás integrantes influye para trabajar con esta nueva metodología que es el aprendizaje cooperativo.

Mediante este análisis descriptivo podemos observar que el cambio de metodología en el aprendizaje del Inglés especialmente el aprendizaje cooperativo si influye positivamente en el proceso. 


\section{Tabla 3}

Análisis de destrezas en Inglés

\begin{tabular}{|c|c|c|c|}
\hline $\begin{array}{l}\text { Unidad de } \\
\text { análisis }\end{array}$ & Categoría & Código & Segmento \\
\hline Reading & $\begin{array}{l}\text { Leer varias veces para entender la investigación } \\
\text { Traducir términos desconocidos }\end{array}$ & $\mathrm{RS}$ & $\begin{array}{l}9 \\
9\end{array}$ \\
\hline Writing & $\begin{array}{l}\text { Apoyarse mutuamente para mejor la escritura. } \\
\text { Buscar información acerca de la escritura en herramientas }\end{array}$ & & $\begin{array}{l}6 \\
9\end{array}$ \\
\hline & $\begin{array}{l}\text { tecnológicas como tutoriales. } \\
\text { Dificultad de traducir lo que se piensa en español al inglés. }\end{array}$ & WS & 9 \\
\hline Listening & $\begin{array}{l}\text { Escuchar atentamente para entender el trabajo. } \\
\text { Escuchar atentamente las ideas de cada integrante. }\end{array}$ & LS & $\begin{array}{l}7 \\
8\end{array}$ \\
\hline Speaking & $\begin{array}{l}\text { Apoyarse en los compañeros que tenían facilidad al } \\
\text { pronunciar palabras. } \\
\text { Mejorar la pronunciación de los verbos en pasado. } \\
\text { Repetir varias veces la grabación hasta }\end{array}$ & SS & $\begin{array}{l}9 \\
9\end{array}$ \\
\hline
\end{tabular}

Fuente: Elaboración propia

Luego para conseguir otra parte del objetivo de esta investigación y evaluar el producto final del aprendizaje cooperativo está plasmado en la tabla 3, en primer lugar se quería realizar rúbricas para demostrar que se potenciaron las destrezas en Inglés pero en realidad no se llevó a cabo debido a que el mundo sufrió emergencia sanitaria y no se efectivizó, en cambio se organizó una entrevista en una sesión de zoom con 9 estudiantes quienes eran coordinadores de los grupos, para indagar que tan efectivo fue la técnica del rompecabezas y la presentación del trabajo en Powtoon para obtener mejorar las destrezas en Inglés a lo que ellos manifestaron que en su mayoría les ayudo a desarrollar sus destrezas ya que las actividades antes mencionadas fueron llevadas cabo dentro del trabajo. 


\section{Tabla 4}

Promedio Evaluación por bloques

\begin{tabular}{llll} 
& Bloque 2 & Bloque 3 & Bloque 4 \\
\hline Segundo BGU "A" & 7.4 & 8.96 & 9.04 \\
Segundo BGU "C" & 7.05 & 8.8 & 9.48 \\
\hline
\end{tabular}

Fuente: Elaboración propia

El siguiente análisis tabla 4 hace referencia a que el aprendizaje cooperativo ayudó a los estudiantes de segundo de bachillerato de la Unidad Educativa "La Providencia" a mejorar su rendimiento especialmente en el cuarto bloque que se inició el 02-02-2020 hasta el 03-05-2020 reflejado de la siguiente manera: entre el bloque tres y cuatro si se observa un mejor aprovechamiento de los estudiantes.

Como se puede deducir luego de estos resultados obtenidos de la investigación que el aprendizaje cooperativo influyo positivamente en los estudiantes del grupo experimental, pero lamentablemente no se logró cumplir con las expectativas de desarrollar más a profundidad las destrezas debido a él gran problema mundial COVID19.

El objetivo de este trabajo investigativo de campo experimental fue de demostrar que el aprendizaje cooperativo influye en un cambio de actitud en los estudiantes frente al proceso de aprendizaje y se lo demostró en las tablas 1 y 2 tal y como lo confirma (ChingYing \& Hui-Yi Wu2, 2013), que el grupo experimental después de un semestre de utilizar este método se observó más el gusto por la asignatura y dedicación.

El trabajo cooperativo no se puede aplicar sin una técnica que en este caso es el rompecabezas que ayuda a los estudiantes a trabajar en grupo, aceptar las ideas de los demás integrantes mediante una interacción y trasmisión de conocimientos con actividades instruidas por el profesor ayuda a potenciar las destrezas del idioma Inglés como lo dice (Namaziandost, Shatalebi, \& Nasri, 2019), quienes afirman que el trabajo cooperativo es importante para potenciar la destreza de hablar y (Hamzah-Alamri, 2018) demuestra que la técnica del rompecabezas en el aprendizaje cooperativo tuvo un resultado significativo en el desarrollo de los estudiantes en la parte de vocabulario.

Al igual la tecnología juega un papel muy importante dentro de la educación no solo en 
Inglés sino en varias disciplinas, en este caso se utilizó la aplicación Powtoon, pero tiene su limitante de que no todas las instituciones educativas poseen un laboratorio, y acceso a internet para desarrollar esta actividad sin embargo hay otras aplicaciones que se pueden utilizar para llegar a potenciar destrezas en inglés.

\section{PROPUESTA}

Este aprendizaje cooperativo tiene su propuesta de trabajar en grupo o pares, mediante las diferentes técnicas como es el rompecabezas Jigsaw, TAl; Team Assisted Indivudualization, la tutoría entre iguales- Peer tutoring, los grupos de investigacióngroup investigación para llegar a conseguir el objetivo del aprendizaje; que los estudiantes sean capaces de crear su propio conocimiento de forma conjunta respetando la heterogeneidad entre ellos.

Una propuesta que se da para los profesores de Inlgés a incentivar en los estudiantes el gusto de aprender este idioma de una forma muy diferente utilizando una metodlogia muy simple que se denomina SAPIE utilizada para organizar el aprendizaje cooperativo, el mismo que encierra todo un proceso para conseguir un objetivo en común.

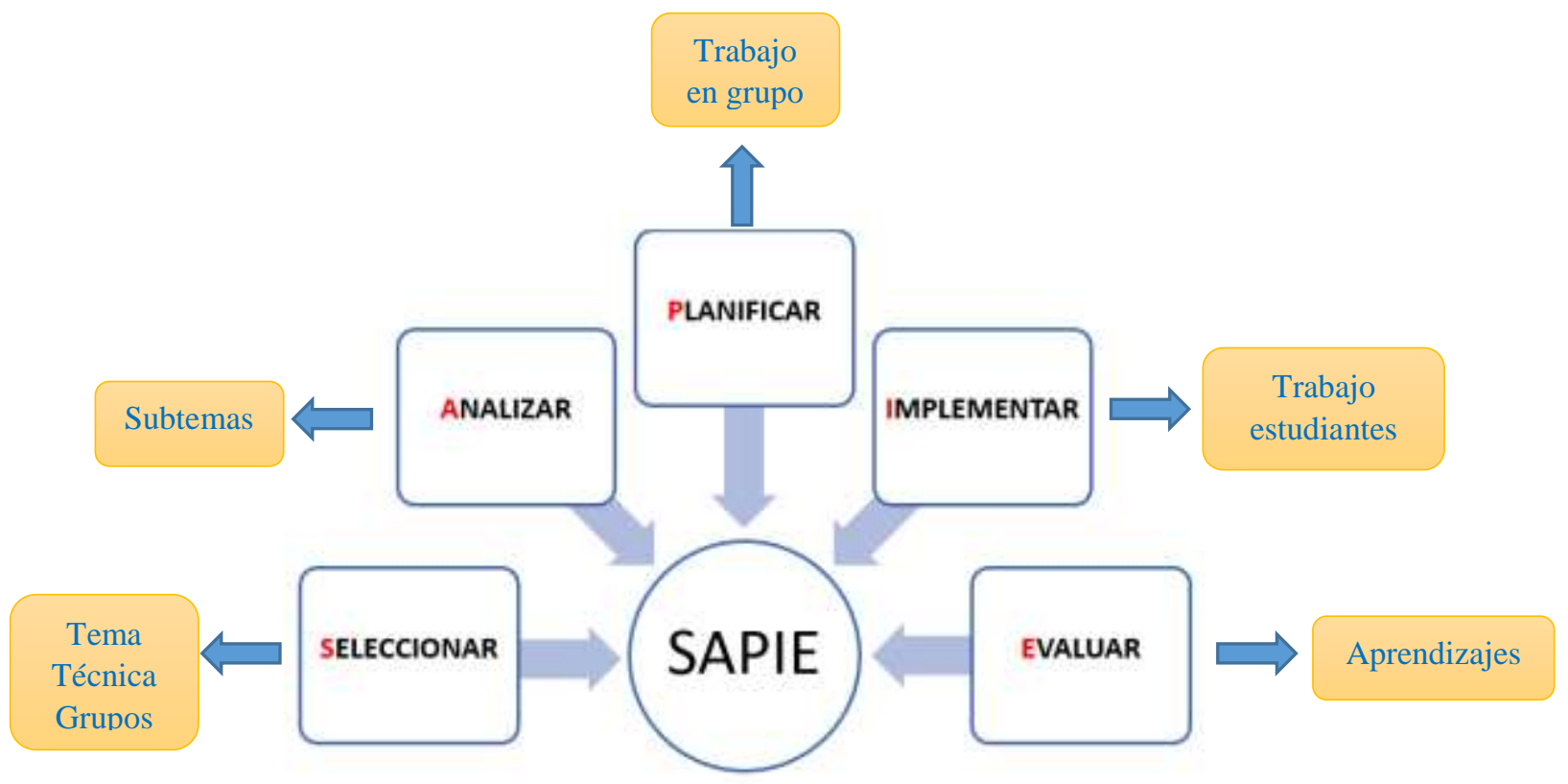

Figura 1. Metodología empleada. 
1.- Seleccionar: en primer lugar la selección de la metodología y su técnica en este caso el aprendizaje coopertativo y su técnica del rompecabezas, luego se realizará la selección del tema principal a trabajar, y por último la selección de grupos. Esta selección es llevada a cabo por el docente quien conoce sus estudaitntes fortalñezas y debilidades, de tal forma que el grupo sea heterogéneopero con las mismas posiblidades para todos. Son grupos de 4 integrantes, quienes están guiados por un coordinador, o lider dentro del grupo quienes les ayuda a desarrollar todas las activdiades.

2.- Analizar: se realizará el análisis cuidadosamente del tema o subtemas que se va a trabajar en clases tomando en cuenta los contenidos de la planificación anual. A demás, las preguntas que cada grupo tendrá que responder en base al tema o subtema tarea que es desdarrollada por el profesor.

3.- Planificar: Esta es una etapa muy importante ya que se organizará el trabajo en grupo, las diferentes actividades que los estudiantes desarrollarán, como el analizar el material investigado, leer conjuntamente para llegar a un consenso de las posibles respuestas a las preguntas dadas por el profesor y crear su propío conocimiento sobre el tema o subtema que se debe trabajar. En estas actividades los estudiantes desarrollarán las destrezas de reading and writing en Inglés. También se establece el tiempo en el que los estudiantes deben presentar las actividades en una semana o dos.

4.- Implmentar: Durante esta fase los estudiantes deben realizar el trabajo en power point, para ello necesitan una computadora por grupo o si el estabalecimiento tiene laboratorio se utilizará para desarrollar esta actividad de tal manera que los estudiantes van creando su propio conocimiento guiados por el profesor. Luego de esta actividad los estudiantes escojerán la aplicación de Powtoon para mejorar su presentación, esta aplicación le facilitara varias opciones para presentar el producto final como es animación y sonido. Cuando los estudiantes terminen estas actividades ellos habrán mejorado las destrezas de listening y speaking ya que deben escucharse, corregir pronunciación y por último grabarse explicando cada diapositiva.

5.- Evaluar: En esta fase de evaluará todo el proceso que se desarrollo para llegar al producto final mediate rúbricas. Después de cada presentación el profesor realizará su 
retroalimentación he irá armando el contenido a estudiar. Con este proceso el profesor y estudiante interactúan activamente creando conocimiento y el libro de Inglés será tomado como un material de apoyo para este proceso enseñanza- aprendizaje.

\section{Tabla 5}

Rúbrica para evaluación expresión oral (speaking) y listening

\begin{tabular}{|c|c|c|c|}
\hline Criterios & $\begin{array}{l}\text { Nivel } 1: \text { ingenuo } \\
0 \text { puntos }\end{array}$ & $\begin{array}{l}\text { Nivel 2: aprendiz } \\
1 \text { punto }\end{array}$ & $\begin{array}{l}\text { Nivel 3: maestría } \\
2 \text { puntos }\end{array}$ \\
\hline Tono de voz & $\begin{array}{l}\text { Los estudiantes no } \\
\text { modulan } \\
\text { apropiadamente y con } \\
\text { fluidez la voz. }\end{array}$ & $\begin{array}{l}\text { Los estudiantes modulan } \\
\text { con dificultad la fluidez. }\end{array}$ & $\begin{array}{l}\text { Los estudiantes modulan } \\
\text { apropiadamente y con } \\
\text { fluidez la voz.. }\end{array}$ \\
\hline Tono de voz & $\begin{array}{l}\text { La comunicación no } \\
\text { fluye con naturalidad. }\end{array}$ & $\begin{array}{l}\text { La comunicación fluye } \\
\text { con poca naturalidad, }\end{array}$ & $\begin{array}{l}\text { La comunicaión fluye con } \\
\text { total naturalidad. }\end{array}$ \\
\hline $\begin{array}{l}\text { Seguridad en la } \\
\text { exposición de } \\
\text { su trabajo }\end{array}$ & $\begin{array}{l}\text { Durante su puesta en } \\
\text { común no expone con } \\
\text { seguridad su trabajo. }\end{array}$ & $\begin{array}{l}\text { Durante su puesta en } \\
\text { común no siempre actúa } \\
\text { con seguridad en la } \\
\text { exposición de su trabajo }\end{array}$ & $\begin{array}{l}\text { En su puesta en común } \\
\text { actúa con seguridad en la } \\
\text { exposición y presentación } \\
\text { del trabajo. }\end{array}$ \\
\hline $\begin{array}{l}\text { Calidad de } \\
\text { presentación }\end{array}$ & $\begin{array}{l}\text { No se demuestra } \\
\text { creatividad en la } \\
\text { persentación. }\end{array}$ & $\begin{array}{l}\text { Poca creatividad de la } \\
\text { presdentación. }\end{array}$ & $\begin{array}{l}\text { Creatividad en la } \\
\text { presentación. }\end{array}$ \\
\hline $\begin{array}{l}\text { Dominio del } \\
\text { tema. }\end{array}$ & $\begin{array}{l}\text { Los estudiantes } \\
\text { demuentran no tener } \\
\text { conocimiento del tema } \\
\text { a tratarse. }\end{array}$ & $\begin{array}{lr}\text { Los } & \text { estudiantes } \\
\text { demuestran } & \text { poco } \\
\text { conocimiento del tema a } \\
\text { tratarse. }\end{array}$ & $\begin{array}{l}\text { Los estudiantes } \\
\text { demuestran un domino } \\
\text { total del conocimiento del } \\
\text { tema a tratarse. }\end{array}$ \\
\hline $\begin{array}{l}\text { Organización } \\
\text { del tema }\end{array}$ & $\begin{array}{l}\text { Los estudiantes no } \\
\text { presentan el trabajo } \\
\text { con orden. }\end{array}$ & $\begin{array}{l}\text { Los estudiantes } \\
\text { presentan el trabajo con } \\
\text { poco orden. }\end{array}$ & $\begin{array}{l}\text { Los estudiantes } \\
\text { presentan el trabajo en } \\
\text { una forma muy } \\
\text { organizada. }\end{array}$ \\
\hline
\end{tabular}

Fuente: Elaboración propia 


\section{Tabla 5}

Rúbrica para evaluación para lectura (reading) and escritura (writing)

\begin{tabular}{|c|c|c|c|}
\hline Criterios & $\begin{array}{c}\text { Nivel 1: ingenuo } \\
0 \text { Points }\end{array}$ & $\begin{array}{c}\text { Nivel 2: aprendiz } \\
1 \text { Point }\end{array}$ & $\begin{array}{c}\text { Nivel 3: maestría } \\
2 \text { Points }\end{array}$ \\
\hline $\begin{array}{l}\text { Obtención de } \\
\text { Información }\end{array}$ & 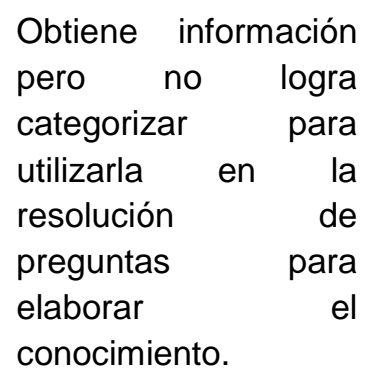 & $\begin{array}{l}\text { Localiza datos e } \\
\text { información importante } \\
\text { dentro del texto. Deja de } \\
\text { lado algunos de ellos en el } \\
\text { momento de resolver } \\
\text { preguntas o no los toma en } \\
\text { cuenta en la elaboración } \\
\text { de otro texto }\end{array}$ & $\begin{array}{l}\text { Localiza información o } \\
\text { una serie de datos de } \\
\text { acuerdo con los } \\
\text { requisitos } \\
\text { características } \\
\text { especificadas en las } \\
\text { preguntas. }\end{array}$ \\
\hline $\begin{array}{l}\text { Comprensión } \\
\text { General }\end{array}$ & $\begin{array}{l}\text { Le cuesta distinguir } \\
\text { las ideas claves o } \\
\text { importantes de un } \\
\text { texto de las ideas } \\
\text { secundarias r y } \\
\text { expresa una idea } \\
\text { vaga y confusa de la } \\
\text { investigación. }\end{array}$ & $\begin{array}{l}\text { Identifica con dificultad las } \\
\text { ideas principales de la } \\
\text { investigación para } \\
\text { organizar correctamente } \\
\text { las ideas. }\end{array}$ & $\begin{array}{l}\text { Identifica las ideas } \\
\text { principales de la } \\
\text { investigación para } \\
\text { organizar } \\
\begin{array}{l}\text { correctamente } \\
\text { ideas. }\end{array}\end{array}$ \\
\hline $\begin{array}{l}\text { Elaboración } \\
\text { De una } \\
\text { Interpretación }\end{array}$ & $\begin{array}{l}\text { Le cuesta entender la } \\
\text { información para ser } \\
\text { procesada e itulizada } \\
\text { para la construcción } \\
\text { del conocimiento. }\end{array}$ & $\begin{array}{l}\text { Identifica con dificultad la } \\
\text { información para ser } \\
\text { procesada e utilizada para } \\
\text { la construcción del } \\
\text { conocimiento. }\end{array}$ & $\begin{array}{lr}\text { Procesa } & \text { la } \\
\text { información para } \\
\text { extraer lo más } \\
\text { importante para la } \\
\begin{array}{l}\text { construcción del } \\
\text { conocimiento. }\end{array}\end{array}$ \\
\hline $\begin{array}{l}\text { Reflexión y } \\
\text { Valoración } \\
\text { Del } \\
\text { Contenido y } \\
\text { La forma }\end{array}$ & $\begin{array}{l}\text { Falta de análisis de } \\
\text { las investigaciones } \\
\text { para organizar mejor } \\
\text { sus apreciaciones y } \\
\text { contenido para la } \\
\text { presentación del } \\
\text { conocimiento. }\end{array}$ & $\begin{array}{l}\text { Analiza con dificultad las } \\
\text { ideas principales ideas de } \\
\text { la investigación y organiza } \\
\text { sus apreciaciones para la } \\
\text { presentación del } \\
\text { conocimiento. }\end{array}$ & $\begin{array}{l}\text { Analiza } \\
\text { correctamente las } \\
\text { ideas de la } \\
\text { investigación para } \\
\text { hacer una excelente } \\
\text { presentación del } \\
\text { conocimiento. }\end{array}$ \\
\hline
\end{tabular}

Fuente: Elaboración propia. 


\section{CONCLUSIONES}

El aprendizaje cooperativo ayuda a desarrollar valores actitudinales al trabajar en grupo y frente a la asignatura del Inglés ya que es un grupo con diferentes habilidades y destrezas, además aceptar las ideas de los demás integrantes les ayudará a fortalecer el valor de la solidaridad, cooperación entre todos. A demás, el aprendizaje cooperativo es una opción muy buena para lograr que los estudiantes mejoren su rendimiento académico en inglés, por la sencilla razón que se involucra las destrezas de una manera implícita en las actividades a desarrollar de tal forma que los estudiantes no se sientan presionados ni nerviosos de ejecutarlas.

La magia de la tecnología logra que los estudiantes exploren y exploten sus habilidades en el manejo de la misma y de esta manera disfruten el aprendizaje llevado a sus clases o laboratorios de diferente manera, son formas innovadoras que deben estas presentes en el desarrollo de las clases ofreciendo muchas opciones para mejora este 'proceso de aprendizaje, una de ellas es: la aplicación Powtoon una herramienta con la que los estudiantes se sienten cómodos para trabajar y mejoren sus destrezas. Esta aplicación diseña otra forma de aprender el idioma Inglés, refuerza sus capacidades de inventar, crear y aplicar su propia creatividad para presentar el trabajo de mejor manera.

Por lo general existen varias técnicas que se pueden aplicar en clases según sea la necesidad del profesor, pero sin embargo la técnica Rompecabezas (Jigsaw) fusionadas con aplicaciones tecnológicas resulta divertido en la enseñanza del idioma Inglés por cuanto les permite crear su propio conocimiento, pero siempre en base a conocimientos previos y de acuerdo a una planificación establecida en la cual exista la interacción del profesor - estudiante para la construcción de un aprendizaje significativo sin olvidar la retroalimentación luego de cada presentación grupal.

En definitiva, hay metodologías activas con sus técnicas, muy favorables a la enseñanza del idioma ingles como es el aprendizaje cooperativo dentro de clases, es hora que los docentes cambien su forma de pensar y aplicarla para lograr varios objetivos como pueden ser: mejorar el rendimiento de los estudiantes, desarrollar destrezas específicas y por ende un cambio en la forma de ver la materia de Inglés sin dejar a lado la tecnología 
Revista Arbitrada Interdisciplinaria KOINONIA

Año 2020. Vol V. ํ⒈ Especial Educación

Hecho el depósito de Ley: FA2016000010 ISSN: 2542-3088

FUNDACIÓN KOINONIA (F.K). Santa Ana de Coro. Venezuela.

Marcia Geoconda González-González; Darwin Gabriel García-Herrera; Nancy Marcela Cárdenas-Cordero; Juan Carlos Erazo-Álvarez

y sus virtudes que tiene en este proceso educativo.

\section{FINANCIAMIENTO}

No monetario.

\section{AGRADECIMIENTO}

A la Universidad Católica de Cuenca por estimular esta investigación de pertinencia social.

\section{REFERENCIAS CONSULTADAS}

Arroyo-Carrera, E., Loor-Santos, M., Mendoza-Mera, J., \& Solorzano-Zambrano, M. (2020). Gestión de aprendizaje creativo mediante la Herramienta Powtoon en estudiantes de lengua y literatura. [Creative learning management through the Powtoon Tool in language and literature students]. EPISTEME KOINONIA, 3(5), 251-267. http://dx.doi.org/10.35381/e.k.v3i5.775

Braun, M. (2017). Comparative Evaluation of Online and In-Class. Journal of University Teaching \& Learning Practice. Obtenido de https://eric.ed.gov/?id=EJ1170210

Briceño-de-Osorio, A. (2019). Aprendizaje de lenguas para la diversidad cultural. [Language learning for cultural diversity]. EPISTEME KOINONIA, 2(4), 5371. http://dx.doi.org/10.35381/e.k.v2i4.524

Borregales, L. (2020). Génesis y evolución socio-histórica de la macro categoría cultura escrita. [Genesis and socio-historical evolution of the macro category written $\begin{array}{llll}\text { culture]. } & \text { EPISTEME }\end{array}$ http://dx.doi.org/10.35381/e.k.v3i5.529

Ching-Ying , P., \& Hui-Yi Wu2, W. (2013). The Cooperative Learning Effects on English Reading Comprehension and Learning Motivation of EFL Freshmen. Canadian Center of Science and Education. Obtenido de https://eric.ed.gov/?id=EJ1076944

Dovale, Y. (2018). Las habilidades resuntivas de ensayos académicos expositivos en estudiantes de la Universidad Nacional Experimental Francisco de Miranda. [The residual skills of expository academic essays in students of the Francisco de Miranda National Experimental University]. EPISTEME KOINONIA, 1(1), 18-34. doi:http://dx.doi.org/10.35381/e.k.v1i1.488 
Fernando, T.-S. (2015). Aprendizaje Cooperativo para la enseñanza de la lengua [Cooperative Learning for language teaching]. Revista Universidad de Granada, 147-162. Obtenido de https://n9.cl/f5uy

FundaciónMapfre. (2020). fundacionmapfre.org. Obtenido de https://n9.cl/txh5

García-Herrera, D., Páez-Quinde, C., Sánchez-Guerrero, J., Infante-Paredes, R., \& Barrera-G , M. (2018). Evaluation of the degree of intellectual maturity in the development of literacy through immersive virtual development of literacy through immersive virtualdevelopment of literacy through immersive virtual worlds: SAPIE methodology. Educon2018, 1354-1359. doi:10.1109 / EDUCON.2018.8363386

García-Sánchez, I. (2015). El aprendizaje cooperativo par el desarrollo de las habilidades sociales en un entorno CLIL [Cooperative learning for the development of social skills in a CLIL environment]. Universidad de Valladolit, Palencia. Obtenido de https://uvadoc.uva.es/handle/10324/15868

Hamzah-Alamri, H. (2018). The Effect of Using the Jigsaw Cooperative Learning Technique on Saudi EFL Students' Speaking Skills. Journal of Education and Practice, 65-77. doi:10.7176 / jep / 45-01-934

lleana de León Sac, M. (junio de 2013). Aprendizaje Cooperativo como estrategia para el aprendizaje del idioma inglés [Cooperative Learning as a strategy for learning the English language]. Universidad Rafael Landívar. Obtenido de https://n9.cl/ud9uq

Kagan, S. (2013). Breve historia de las Estructuras Kagan [Brief history of the Kagan Structures]. Kagan Online Magazine. Obtenido de https://n9.cl/q4dau

Lemos-Rodriguez, R. (2018). El aprendizaje cooperativo como herramienta para la educación universitaria [Cooperative learning as a tool for university education]. Educación en valores. Obtenido de https://n9.cl/3ywy

Loor-Salmon, L., Palma-Villavicencio, M., Saltos-Rodriguez, L., \& Bolívar-Chavez, O. (2018). El aprendizaje cooperativo como una estrategia de enseñanza del Idioma de Ingles en las Escuelas públicas del Ecuador [Cooperative learning as an English language teaching strategy in Ecuador's public schools]. Revista Científica, 431448. doi:http://dx.doi.org/10.23857/dc.v4i3.817 
Martínez-Lirola, M. (2017). Propuesta para desarrollar competencias sociales a través del aprendizaje cooperativo en clases de inglés como lengua extranjera [Proposal to develop social skills through cooperative learning in English as a foreign language classes]. Revista Electrónica Interuniversitaria de portacion del profesorado. doi:https://doi.org/10.6018/reifop/20.1.260021

Namaziandost, E., Shatalebi, V., \& Nasri, M. (2019). The Impact of Cooperative Learning on Developing Speaking Ability and Motivation Toward Learning English. ResearchGate, 83-101. doi:10.17323 / jle.2019.9809

Pujolàs Maset, Pere. (2003). El aprendizaje cooperativo: algunas ideas prácticas [Cooperative learning: some practical ideas]. Unisersidad de Vic., España. Obtenido de https://n9.cl/hgt3

Robayo-Guerrero, J. O. (2019). Some contributionsto English oral interaction from cooperative learning. RHS Revista Humanismo y Sociedad, 22-36. Obtenido de https://n9.cl/n15g

Rusyda, S. A., \& Supari, M. (2019). The Effectiveness of the Cooperative Learning Model of Jigsaw Type. Atlantis Press, 223-229. doi:https://doi.org/10.2991/assehr.k.191217.037

Siguenza-Peñafiel, K. M., Erazo-Álvarez, J. C., \& Narváez-Zurita, C. I. (2020). Estrategias de marketing viral y el posicionamiento de marca en el sector farmacéutico [Viral marketing strategies and brand positioning in the pharmaceutical sector]. Revista Arbitrada Interdisciplinaria Koinonía, 313-338. doi:http://dx.doi.org/10.35381/r.k.v5i10.697

SourceForge. (2020). SourceForge. Obtenido de https://n9.cl/g3rf

Sung, Y., Chang, K., \& Liu, T. (2016). The effects of integrating mobile devices with teaching and learning on students' learning performance: A meta-analysis and research synthesis. Computers \& Education, 252-275. doi:https://doi.org/10.1016/j.compedu.2015.11.008

Torres-Cajas, M. (2017). Metodología cooperativa utilizando las tecnologías de la información y comunicación en el aprendizaje del idioma inglés en los estudiantes de tercer nivel de la Facultad de Ingeniería [Cooperative methodology using information and communication technolog]. Universidad Nacional del Chimborazo, Riobamba. Obtenido de https://n9.cl/2rp67

Universa (2020). Docentes: cómo utilizar la técnica de rompecabezas en el aula [Teachers: how to use the puzzle technique in the classroom]. Obtenido de https://n9.cl//8gs 
Revista Arbitrada Interdisciplinaria KOINONIA

Año 2020. Vol V. ํ⒈ Especial Educación

Hecho el depósito de Ley: FA2016000010

ISSN: 2542-3088

FUNDACIÓN KOINONIA (F.K). Santa Ana de Coro. Venezuela.

Marcia Geoconda González-González; Darwin Gabriel García-Herrera; Nancy Marcela Cárdenas-Cordero; Juan Carlos Erazo-Álvarez

(C)2020 por los autores. Este artículo es de acceso abierto y distribuido según los términos y condiciones de la licencia Creative Commons Atribución-NoComercial-CompartirIgual 4.0 Internacional (CC BY-NC-SA 4.0)

(https://creativecommons.org/licenses/by-nc-sa/4.0/). 